\title{
High-Speed, Low Drive-Voltage Silicon-Organic Hybrid Modulator Based on a Binary-Chromophore Electro-Optic Material
}

\author{
Robert Palmer, Sebastian Koeber, Delwin L. Elder, Markus Woessner, Wolfgang Heni, Dietmar Korn, \\ Matthias Lauermann, Wim Bogaerts, Member, IEEE, Member, OSA, \\ Larry Dalton, Senior Member, IEEE, Member, OSA, Wolfgang Freude, Senior Member, IEEE, Member, OSA, \\ Juerg Leuthold, Fellow, IEEE, Fellow, OSA, and Christian Koos, Member, IEEE
}

(Invited Paper)

\begin{abstract}
We report on the hybrid integration of silicon-oninsulator slot waveguides with organic electro-optic materials. We investigate and compare a polymer composite, a dendron-based material, and a binary-chromophore organic glass (BCOG). A record-high in-device electro-optic coefficient of $230 \mathrm{pm} / \mathrm{V}$ is found for the BCOG approach resulting in silicon-organic hybrid MachZehnder modulators that feature low $U_{\pi} L$-products of down to $0.52 \mathrm{Vmm}$ and support data rates of up to $40 \mathrm{Gbit} / \mathrm{s}$.
\end{abstract}

Index Terms-Electro-optic modulators, nonlinear optical devices, photonic integrated circuits.

\section{INTRODUCTION}

$\mathbf{S}$ ILICON electro-optic modulators are key building blocks of highly integrated photonic circuits and particularly important for future high-performance optical interconnects. Due to the absence of linear electro-optic (EO) effects in bulk sil-

Manuscript received February 3, 2014; revised March 28, 2014; accepted April 30, 2014. Date of publication May 29, 2014; date of current version July 25, 2014. This work was supported by the European Research Council (ERC Starting Grant "EnTeraPIC," number 280145), by the Alfried Krupp von Bohlen und Halbach Foundation, by the Initiative and Networking Fund of the Helmholtz Association, by the DFG Center for Functional Nanostructures (CFN), by the Karlsruhe International Research School on Teratronics (HIRST), by the Karlsruhe School of Optics and Photonics (KSOP), by the Karlsruhe Nano and Micro Facility (KNMF), by the EU-FP7 projects SOFI, OTONES, PHOXTROT, and BigPIPES. This work was also supported by the National Science Foundation (DMR-0905686, DMR-0120967) and the Air Force Offic of Scientifi Research (FA9550-09-1-0682).

R. Palmer, M. Woessner, D. Korn, and M. Lauermann are with the Institute of Photonics and Quantum Electronics, Karlsruhe Institute of Technology, 76131 Karlsruhe, Germany (e-mail: robert.palmer@kit.edu; markus.woessner@ student.kit.edu; korn@kit.edu; matthias.lauermann@kit.edu).

S. Koeber, W. Freude, and C. Koos are with the Institute of Photonics and Quantum Electronics and the Institute of Microstructure Technology, Karlsruhe Institute of Technology, 76131 Karlsruhe, Germany (e-mail: sebastian.koeber@kit.edu; wolfgang.freude@kit.edu; christian.koos@kit.edu).

W. Heni and J. Leuthold were with the Karlsruhe Institute of Technology, 76131 Karlsruhe, Germany. They are now with the Institute of Electromagnetic Fields, ETH Zurich, 8092 Zurich, Switzerland (e-mail: wheni@ethz.ch; JuergLeuthold@ethz.ch).

D. L. Elder and L. Dalton are with the Department of Chemistry, University of Washington, Seattle, WA 98195-1700 USA (e-mail: elderdl@uw.edu; dalton@chem.washington.edu).

W. Bogaerts is with the Ghent University - IMEC, 9000 Gent, Belgium. He is now with Luceda Photonics, 9200 Dendermonde, Belgium (e-mail: wim.bogaerts@ugent.be).

Color versions of one or more of the f gures in this paper are available online at http://ieeexplore.ieee.org.

Digital Object Identifie 10.1109/JLT.2014.2321498 icon, current modulators mostly rely on the plasma-effect, using either free-carrier depletion [1] or injection [2] in diode or metal-oxide-semiconductor-(MOS-)structures [3]. However, trade-offs have to be made when aiming at fast devices that feature low drive voltage and small footprint simultaneously. In particular, carrier-injection devices feature voltage-length products as small as $U_{\pi} L=0.36 \mathrm{Vmm}$, but free-carrier lifetime limits modulation speed to few Gbit/s if no pre-emphasis of the drive signal is used [2]. In contrast, carrier-depletion modulators support symbol rates of up to $50 \mathrm{GBd}$ [4], but typical voltage-length products are beyond $10 \mathrm{Vmm} \mathrm{[5],} \mathrm{[6]} \mathrm{and} \mathrm{thus}$ much larger than those of injection-type devices. Drive voltage and device footprint can be strongly reduced by using resonant structures [5], [7]. However, resonant devices feature limited optical bandwidth and their resonance wavelength is particularly prone to temperature fluctuations

Silicon-organic hybrid $(\mathrm{SOH})$ integration pursues a fundamentally different approach [8], [9]. Here, light is guided in a silicon waveguide core and nonlinear optical effects of second order are realized by exploiting evanescent interaction of the guided light mode with an organic electro-optic cladding material [10]-[12]. Specificall tailored organic materials with strong linear EO effect (Pockels effect) enable small voltagelength products and high modulation bandwidth simultaneously [10], [13]-[15]. So far, the most commonly used cladding materials for SOH integration are polymers doped by $\mathrm{EO}$ chromophore molecules [11], [16]. While these guest-host materials have shown EO coefficient as high as $118 \mathrm{pm} / \mathrm{V}$ in bulk material [17], values measured in $\mathrm{SOH}$ devices were much smaller, ranging from 20 to $60 \mathrm{pm} / \mathrm{V}$ [11], [16], [12], [18]-[20]. This has significantl limited the performance of $\mathrm{SOH}$ electro-optic modulators up to now.

In this paper we demonstrate that in-device EO coefficient can be significantl increased by using a binary electro-optic material system consisting of shape-engineered spherical dendritic and rod-shaped dipolar chromophores. This material system clearly outperforms conventional guest-host and neat dendritic materials. We demonstrate a record-high in-device EO coeffi cient of $r_{33}=230 \mathrm{pm} / \mathrm{V}$, which results in a low modulator voltage-length product of $U_{\pi} L=0.52 \mathrm{Vmm}$. In addition we show that these modulators support data rates of up to $40 \mathrm{Gbit} / \mathrm{s}$. 

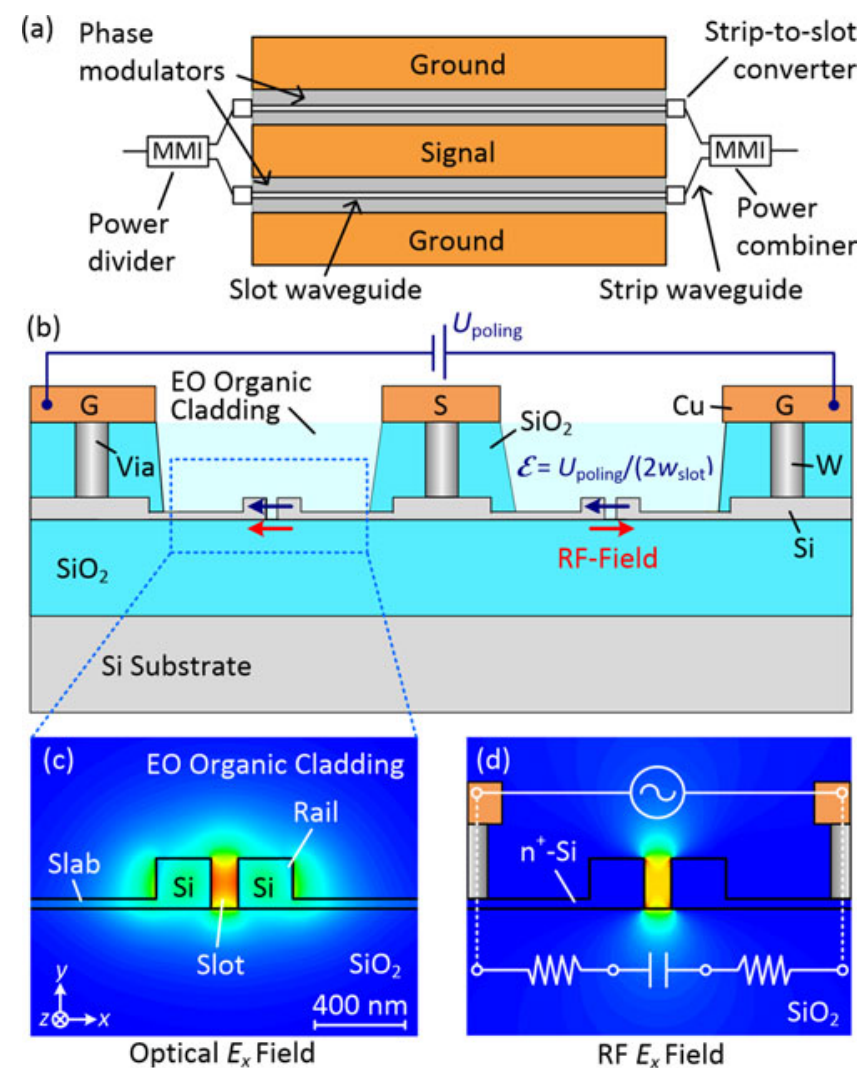

Fig. 1. SOH MZM. (a) Schematic of the MZM. The device consists of two slot waveguide phase modulators, driven in push-pull operation by a single coplanar ground-signal-ground (GSG) transmission line. Multimode interference couplers (MMI) are used as power splitters and combiners. Logarithmically tapered low-loss strip-to-slot mode converters are used for coupling access strip waveguides to slot waveguides [23]. (b) Cross-section of the MZM. The organic material is poled by applying a poling voltage $U_{\text {p oling }}$ across the float ing ground electrodes of the modulator. Dark blue arrows denote the orientation of the chromophores after poling. The red arrows indicate the orientation of the RF modulation fiel of the GSG transmission line, which is parallel to the chromophore orientation in the left phase shifter and anti-parallel in the phase shifter on the right-hand side, thereby resulting in push-pull operation of the device. (c) Cross-sectional view and simulated optical mode of one phase modulator. The light is strongly confine to the slot region due to electric-fiel discontinuities at the slot sidewalls. (d) Simulated RF mode fiel of the slot waveguide. The two rails of the silicon slot waveguide are electrically connected to metal electrodes by $60 \mathrm{~nm}$-high n-doped (As, $7 \times 10^{17} \mathrm{~cm}^{-3}$ ) silicon slabs. The modulation voltage drops across the narrow slot resulting in a high modulation fiel that has a strong overlap with the optical mode.

\section{Silicon-Organic Hybrid MOdUlator}

Our experiments are based on single-drive SOH MachZehnder modulators (MZM), see Fig. 1(a) for an illustration of the device structure. The MZM comprises two SOH phasemodulators that are driven in push-pull mode by a single coplanar transmission line in ground-signal-ground configuratio (GSG). Each of the phase modulators consists of a slot waveguide (slot width $160 \mathrm{~nm}$, rail width $210 \mathrm{~nm}$ ), which is covered by an organic EO material, Fig. 1(b). The fundamental optical quasi-TE mode fiel of the waveguide is strongly confine to the slot region due to fiel discontinuities at the slot sidewalls [21], Fig. 1(c). At the same time, the copper strips of the transmission line are electrically connected to the rails of the phase modulators by $900 \mathrm{~nm}$-high tungsten vias and $60 \mathrm{~nm}$-thick n-doped (as, $7 \times 10^{17} \mathrm{~cm}^{-3}$ ) silicon slabs. A voltage applied to the transmission line drops across the narrow slot, resulting in a high modulation fiel that strongly overlaps with the optical quasi-TE mode, see Fig. 1(d).

The waveguide structures were fabricated by $193 \mathrm{~nm}$ deepUV lithography on an 8" silicon-on-insulator wafer with $220 \mathrm{~nm}$ device layer thickness and $2 \mu \mathrm{m}$-thick buried oxide as described in [22]. The fabrication of the silicon photonic integrated circuit (PIC) was carried out using a combination of CMOS-based technology and deposition of the electro-optic cladding in a postprocessing step. The transmission line is composed of tungsten vias, an oxide buffer layer, and copper electrodes [22]. After fabrication the oxide cladding is locally removed in the slot waveguide regions by a combination of dry and wet etching to open the slots for applying the EO organic material. The EO organic material is then deposited from a solution by spincoating and fill the opened oxide regions and the slots of the slot waveguides. Deposition of the electro-optic material can hence be completely decoupled from CMOS processing.

Directly after the deposition, the organic material does not feature any macroscopic EO effect due to the random orientation of the chromophore molecules. For inducing macroscopic EO activity the material needs to be poled. This is achieved by heating the sample to the glass-transition temperature $T_{g}$ of the organic material while applying a dc poling voltage $U_{\text {poling }}$ between the floatin ground electrodes. The resulting poling fiel in the slot aligns the dipolar chromophores in the slot as indicated by the dark blue arrows in Fig. 1(b) [13]. While holding the poling voltage, the chip is cooled back to room temperature, thereby conserving the acentric order of the chromophores. The RF modulation fiel of the GSG transmission line, indicated by red arrows in Fig. 1(b), is parallel to the chromophore orientation in the left phase shifter and anti-parallel in the phase shifter on the right-hand side. This results in push-pull operation of the device

In the following, we investigate two SOH MZM of different lengths: A $1 \mathrm{~mm}$-long modulator that is terminated by a $50 \Omega$ load, and a $250 \mu \mathrm{m}$-long modulator without termination ("lumped"). Grating couplers are used for fibe -chip coupling [24]. The fibe -to-fibe loss of a $1 \mathrm{~mm}$-long MZM is $16.5 \mathrm{~dB}$, dominated by fibe -chip coupling losses of the non-optimized grating couplers. The on-chip loss of the MZM amounts to approximately $6 \mathrm{~dB}$ for maximum transmission of the modulator, which can be decomposed into losses of passive components, material absorption loss of the organic cladding, free-carrier absorption within the doped silicon waveguides, and scattering loss due to sidewall roughness of the slot waveguide. Passive components, such as MMI and strip-to-slot mode converters contribute only $0.5 \mathrm{~dB}$ to the total loss. Material absorption of the organic cladding amounts to $0.2 \mathrm{~dB} / \mathrm{cm}$ in the C-band and can be neglected. Free-carrier absorption and scattering loss hence remain the dominant effects. For the free-carrier absorption, we estimate a contribution of approximately $1.3 \mathrm{~dB} / \mathrm{cm}$, taking into account that only around $50 \%$ of the guided light actually interacts with the doped silicon structure, in which the donor doping concentration amounts to $7 \times 10^{17} \mathrm{~cm}^{-3}$ [25]. The 
remaining propagation losses of $4 \mathrm{~dB} / \mathrm{mm}$ are attributed to scattering due to fabrication imperfections that occur during etching of the waveguides and during opening of the back-end oxide. We expect that scattering losses can be significantl reduced by optimization of the process parameters. For $300 \mathrm{~mm}$ CMOS technology, recent developments [26] indicate that the propagation loss of slot waveguides can be below $1 \mathrm{~dB} / \mathrm{mm}$. Similarly, for $200 \mathrm{~mm}$ technology, propagation losses below $0.7 \mathrm{~dB} / \mathrm{mm}$ have been reported using larger slots of $190 \mathrm{~nm}$ width [27], and these figure can be further reduced by deploying asymmetric slot geometries, where losses of $0.2 \mathrm{~dB} / \mathrm{mm}$ have been demonstrated [28]. We believe that an optimization of the fabrication process and a reduction of the device length will enable nextgenerations of SOH MZM that feature an insertion loss of 1 to $2 \mathrm{~dB}$.

\section{ORGANIC EleCtro-OPtic MATERIALs AND POLING}

A widely used $\mathrm{EO}$ material class for $\mathrm{SOH}$ integration are chromophore-polymer guest-host materials. The concentration of the EO active chromophores in these materials is typically limited to $25 \mathrm{wt} . \%$ to prevent detrimental head-to-tail orientation of the dipolar molecules and partial crystallization of the material. The efficien activation of the second-order nonlinearity in $\mathrm{SOH}$ devices remained an unsolved problem and reported in-device EO coefficient of these materials were a factor 2 to 5 below the corresponding values of bulk organic materials [11], [12], [16]-[20]. This phenomenon is usually explained by incomplete poling.

It has recently been shown that in-device EO coefficient of up to $r_{33}=180 \mathrm{pm} / \mathrm{V}$ can be achieved by using so-called monolithic EO materials, that do not require a polymer matrix to prevent detrimental dipole-dipole interaction and that have a molecular structure that is engineered for enhanced poling efficien y [15], [17]. Here we expand on these finding by investigating two different material systems: A neat material that consists of the multi-chromophore dendritic molecule PSLD41 [29], and a mixture of the chromophores YLD124 and PSLD41 (25:75 wt.\%), also referred to as binary chromophore organic glass (BCOG) [30]. These materials are compared to a conventional guest-host material consisting of YLD124 (25 wt.\%) in a PMMA matrix [17]. The chemical structures of the involved chromophores are depicted in Fig. 2. The chromophore YLD124, Fig. 2(a), is a simple rod-shaped EO molecule that only consists of a donor and an acceptor group, linked by a ring-locked $\pi$-conjugated bridge. The dendritic chromophore PSLD41, Fig. 2(b), consists of a central connecting motif to which three EO substructures are attached (marked in green). This results in a spherical shape of the molecule, which is known to lead to an improved poling efficien y as compared to oblate and prolate structures [29], [31]. In addition, perfluoraryl containing side-groups (marked in blue) are attached to the EO substructures. These site-isolation groups effectively reduce the dipole-dipole interaction of neighboring molecules [31]. This way, PSLD41 can be used as a neat material without the need of an isolating polymer matrix.
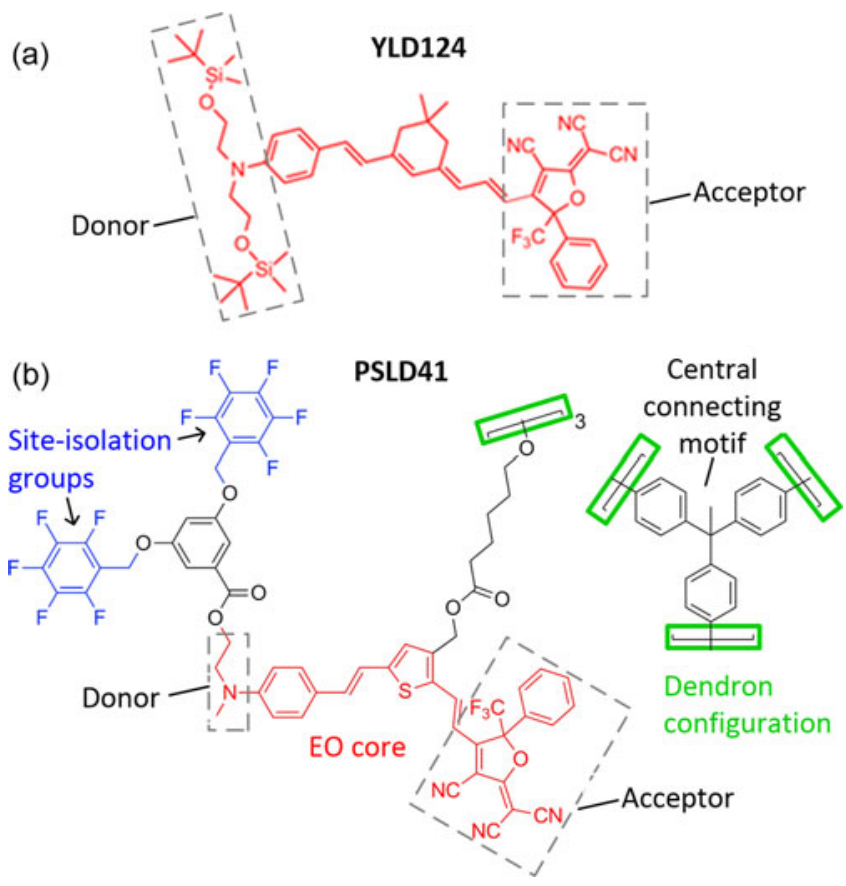

Fig. 2. Chemical structures of the individual chromophores. The EO cores of the chromophores are drawn in red. (a) The chromophore YLD124 consists only of a donor group, of an acceptor group, and of a $\pi$-conjugated bridge. (b) PSLD41 is a dendritic configuratio that combines three EO substructures, marked in green. Each EO substructure comprises one electro-optic core with functional sidegroups (blue) that lead to a define three-dimensional dendron structure with spherical shape. The site-isolation groups (blue) decrease intermolecular interaction and thereby reduce head-to-tail orientation of neighboring molecules, while the spherical shape results in an enhancement of poling effi ciency [32]

Of special interest is the investigation of the BCOG composed of $25 \mathrm{wt} . \%$ YLD124 and $75 \mathrm{wt} . \%$ PSLD41. It has been shown that the EO activity of a BCOG is greater than the sum of its individual components [30]. Formation of a stable YLD124/PSLD41-complex during poling was offered as an explanation for this remarkable phenomenon [30].

For comparison, the three materials are applied to nominally identical chips by spin-coating from an $8 \%$ solution dissolved in 1,1,2-trichloroethane. After deposition, the materials are poled for push-pull operation of the MZM by applying a poling voltage between the floatin ground electrodes of the modulator, as depicted in Fig. 1(b). After poling, the $\pi$-voltage $U_{\pi}$ of the modulator is derived from the voltage-dependent transmission characteristics, see Fig. 3. For better comparison of the EO coefficients the $\pi$-voltage is measured at a dc bias $>5 \mathrm{~V}$, since free charges in the cladding lead to partial fiel screening at small dc fields However, this effect is slow and does not impair the modulation efficien $y$ of our device for frequencies $>1 \mathrm{kHz}$, i.e., the $\pi$-voltage is found to be independent of the bias voltage at frequencies $>1 \mathrm{kHz}$.

The EO coefficien $r_{33}$ can be obtained from the measured $\pi$ voltage $U_{\pi}$ of the push-pull MZM by using the relation [10], [33]

$$
U_{\pi}=\frac{w_{\text {slot }} \lambda_{\mathrm{c}}}{2 L r_{33} \Gamma n_{\text {slot }}^{3}} .
$$



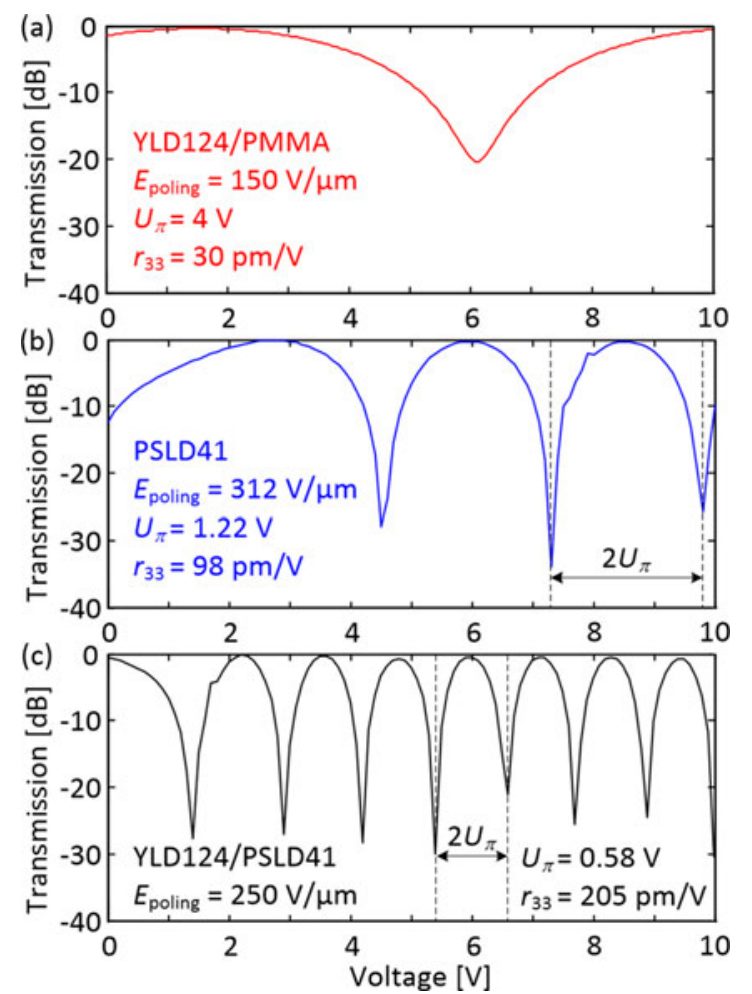

Fig. 3. Voltage-dependent transmission of $1 \mathrm{~mm}$-long SOH MZM coated with different organic materials. The respective poling field $E_{\text {poling }}$, measured $\pi$-voltages $U_{\pi}$, and derived EO coefficient $r_{33}$ are specifie in the respective figures (a) YLD124/PMMA cladding. (b) PSLD41 cladding. (c) YLD124/PSLD41 cladding. The smallest $U_{\pi}$ is found for the BCOG YLD124/PSLD41. The $\pi$-voltage is measured at a dc bias $>5 \mathrm{~V}$ to remove the effect of free charges in the cladding, that lead to partial fiel screening at small dc fields

The quantity $w_{\text {slot }}$ denotes the measured slot width, $\lambda_{\mathrm{c}}$ is the wavelength of the optical carrier, $\mathrm{L}$ is the modulator length, $n_{\text {slot }}$ is the refractive index of the organic material in the slot, and $\Gamma$ is the fiel interaction factor that is linked to the fraction of the optical power that interacts with the modulation fiel [10], [33]

$$
\Gamma=\frac{n_{\text {slot }}}{Z_{0}} \frac{\int_{A_{\text {slot }}}\left|\mathcal{E}_{x}\right|^{2} d A}{2 \mathcal{P}\left(\omega_{c}\right)} .
$$

Here $\mathcal{E}_{x}$ is the $x$-component of the electric fiel of the optical mode, $\mathcal{P}$ is the power of the optical mode field $Z_{0}$ is the freespace wave impedance, and $A_{\text {slot }}$ is the cross-sectional area of the silicon slot. For the device geometry used in our experiments, the calculated fiel interaction factors of the three investigated materials differ by less than $2 \%$ and amount to $\Gamma \approx 0.2$.

\section{RESUlts OF THE POLING EXPERIMENTS}

For each of the investigated materials, we perform poling experiments at various poling field $E_{\text {poling }}$. The resulting EO coefficient $r_{33}$ are depicted in Fig. 4.

\section{A. Guest-Host System}

First, the guest-host material YLD124/PMMA is investigated. The highest observed EO coefficien is $30 \mathrm{pm} / \mathrm{V}$ and was achieved by applying a poling fiel of $150 \mathrm{~V} / \mu \mathrm{m}$. The

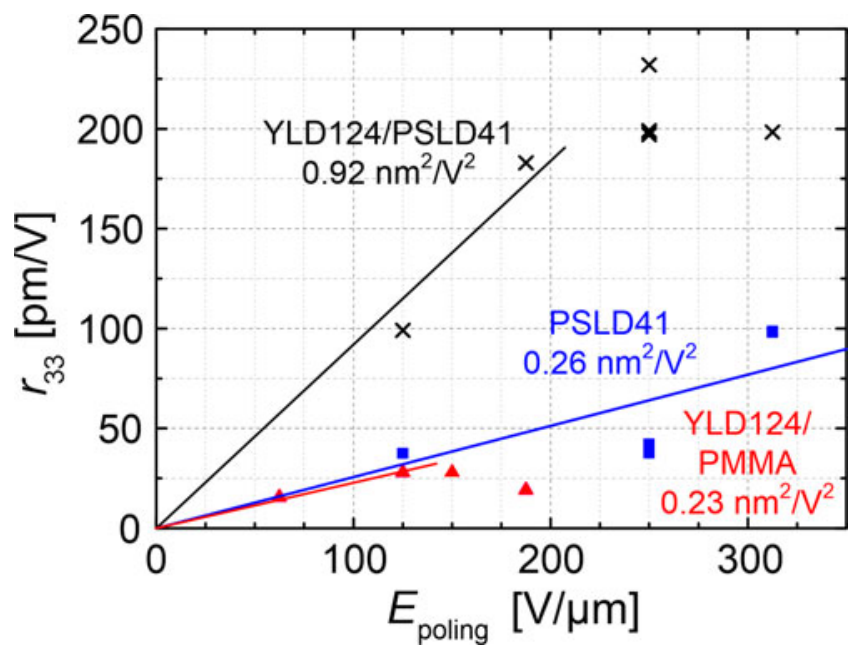

Fig. 4. Measured poling efficiencie $r_{33} / E_{\text {poling }}$ for three different organic cladding materials: PMMA doped with 25 wt.\% YLD124 (guest-host system, red), the neat dendritic chromophore PSLD41 (blue), and PSLD41 doped by 25 wt.\% YLD124 (binary chromophore organic glass, black). The solid lines represent linear fit in the limit of low poling fields The slope of these lines is used as a measure for poling efficien y. For the YLD124/PMMA mixture, only data points at low poling field $\left(E_{\text {poling }}<150 \mathrm{~V} / \mu \mathrm{m}\right)$ were considered for the fit

corresponding voltage-dependent transmission of a $1 \mathrm{~mm}$-long device is depicted in Fig. 3(a). The $\pi$-voltage is $U_{\pi}=4 \mathrm{~V}$. When applying higher poling field the measured EO coefficien decreases, see Fig. 4. This is attributed to an increase of material conductivity during poling at high fiel strength. For low poling fields a linear relation between poling fiel and EO coefficien is expected [29], whereas for poling field beyond $200 \mathrm{~V} / \mu \mathrm{m}$, dielectric breakdown occurs. By linear regression of the data points obtained for low poling field $E_{\text {poling }}<150 \mathrm{~V} / \mu \mathrm{m}$ (red line in Fig. 4) we determine a poling efficien y $r_{33} / E_{\text {poling }}$ of $0.23 \mathrm{~nm}^{2} / \mathrm{V}^{2}$. The measured in-device $\mathrm{EO}$ coefficient and the measured poling efficiencie are far lower than values reported in parallel-plate poled bulk reference samples, where maximum electro-optic coefficient of $r_{33, \max }=118 \mathrm{pm} / \mathrm{V}$ and poling efficiencie of $r_{33} / E_{\text {poling }}=1.27 \mathrm{~nm}^{2} / \mathrm{V}^{2}$ were achieved [17]. These finding are thus in good agreement with finding in other publications where poling of similar guest-host materials in $\mathrm{SOH}$ devices was found to be inefficien [11], [12], [16], [18]-[20].

\section{B. Multi-Chromophore Dendritic Molecules}

Next, we analyze a sample coated with neat PSLD41 [29]. We fin a slightly higher poling efficien y $r_{33} / E_{\text {poling }}$ of $0.26 \mathrm{~nm}^{2} / \mathrm{V}^{2}$ (blue line in Fig. 4) and a remarkably high stability of the material against dielectric breakdown. For the highest applied poling fiel of $312 \mathrm{~V} / \mu \mathrm{m}$, we fin an EO coefficien of $r_{33}=98 \mathrm{pm} / \mathrm{V}$. The voltage-dependent transmission of the MZM is depicted in Fig. 3(b) and we extract $U_{\pi}=1.22 \mathrm{~V}$. Note that for this material, the achieved in device EO coefficien $r_{33}$ is even higher than its bulk-material counterpart: In bulk reference samples, EO coefficient of $r_{33}=90 \mathrm{pm} / \mathrm{V}$ were measured at a poling fiel of $E_{\text {poling }}=90 \mathrm{~V} / \mu \mathrm{m}$ [31]. 
TABLE I

Summary of Measured EO Coefficients. Comparison Between in-Device Values and Values Achieved in Parallel-Plate Poled Bulk Reference SAmples. For Poling, the SAmples Were Heated to the Glass Transition Temperature $T_{g}$

\begin{tabular}{|c|c|c|c|c|c|c|c|c|}
\hline \multirow[b]{2}{*}{ Material } & \multirow[b]{2}{*}{$n$} & \multicolumn{2}{|c|}{ Bulk material } & \multicolumn{3}{|c|}{ In-device } & \multirow[b]{2}{*}{$\begin{array}{c}U_{\pi} \boldsymbol{L} \\
{[\mathrm{Vmm}]}\end{array}$} & \multirow[b]{2}{*}{$T_{\mathrm{g}}\left[^{\circ} \mathrm{C}\right]$} \\
\hline & & $\begin{array}{c}r_{33} \\
{[\mathrm{pm} / \mathrm{V}]}\end{array}$ & $\begin{array}{l}r_{33} / E_{\text {poling }} \\
{\left[\mathrm{nm}^{2} / \mathbf{V}^{2}\right]}\end{array}$ & $\begin{array}{c}r_{33} \\
{[\mathrm{pm} / \mathrm{V}]}\end{array}$ & $\begin{array}{l}r_{33} / E_{\text {poling }} \\
{\left[\mathrm{nm}^{2} / \mathbf{V}^{2}\right]}\end{array}$ & $\begin{array}{c}n^{3} r_{33} \\
{[\mathrm{pm} / \mathrm{V}]}\end{array}$ & & \\
\hline YLD124(25wt.\%)/PMMA & 1.7 & $118[17]$ & $1.27[17]$ & 30 & 0.23 & 147 & 4 & 105 \\
\hline PSLD41 & 1.72 & $90[31]$ & $1.04[31]$ & 98 & 0.26 & 499 & 1.22 & 103 \\
\hline YLD124(25wt.\%)/PSLD41 & 1.73 & $285[30]$ & $2.85[30]$ & 230 & 0.92 & 1190 & 0.52 & 97 \\
\hline
\end{tabular}

Even though this corresponds to a much higher poling efficien y of $r_{33} / E_{\text {poling }}=1.04 \mathrm{~nm}^{2} / V^{2}$, the achievable EO coefficien is smaller due to the early onset of dielectric breakdown, which limits the maximum applicable poling fiel to $\sim 100 \mathrm{~V} / \mu \mathrm{m}$ in bulk PSLD41.

\section{Binary Chromophore Organic Glass}

Finally, we investigate the mixture of 25wt.\% YLD124 and $75 \mathrm{wt} . \%$ PSLD41 on a slot waveguide sample. We fin a remarkably high poling efficien y of $r_{33} / E_{\text {poling }}=0.92 \mathrm{~nm}^{2} / \mathrm{V}^{2}$ $\left(E_{\text {poling }}<200 \mathrm{~V} / \mu \mathrm{m}\right.$, black line in Fig. 4). Again, we observe excellent stability of the material against dielectric breakdown. Above a poling fiel of $250 \mathrm{~V} / \mu \mathrm{m}$, the achieved EO coefficien appears to saturate to a value of $\sim 200 \mathrm{pm} / \mathrm{V}$. Fig. 3(c) shows the voltage-dependent transmission behavior of a $1 \mathrm{~mm}$-long MZM. The $\pi$-voltage is $U_{\pi}=0.58 \mathrm{~V}$. In the best case we achieved an EO coefficien of $r_{33}=230 \mathrm{pm} / \mathrm{V}\left(U_{\pi} L=0.52 \mathrm{Vmm}\right)$, by poling with a fiel strength of $250 \mathrm{~V} / \mu \mathrm{m}$. This is the highest reported $\mathrm{EO}$ coefficien in an $\mathrm{SOH}$ device, and it even exceeds the previously reported record value of a fully organic MZM, where $137 \mathrm{pm} / \mathrm{V}$ were measured [34]. Note that a significantl larger poling efficien y is observed in the bulk BCOG $\left(2.85 \mathrm{~nm}^{2} / \mathrm{V}^{2}\right)$, but also here, low in-device poling efficien y is compensated by the fact that higher poling field can be applied in the slot waveguide as compared to the bulk material. The observed increase in poling efficien $y$ of the BCOG as compared to the neat PSLD41 and to YLD124 in PMMA is in good agreement with results obtained in bulk material, where the binary chromophore system YLD124/PSLD41 was found to have an $r_{33}$ coefficien (285 $\mathrm{pm} / \mathrm{V}$ ) that exceeds even the sum of the $r_{33}$ coefficient of its constituents [30].

\section{Discussion}

A summary of the poling results is shown in Table I. From the experiments, we can conclude that the in-device poling efficien cies of all three investigated organic materials are a factor 3-4 smaller than the values obtained for the respective bulk samples. Nevertheless, the achievable in-device $r_{33}$-coefficien may be very close to or even higher than the bulk-material reference when using structurally engineered chromophores as PSLD41. This is enabled by enhanced material stability against dielectric breakdown in $\mathrm{SOH}$ slot waveguides as compared to bulk references, which allows to apply stronger poling fields We attribute this to thin fil effects and to a low number of defects in the slot region. An interesting findin of this material comparison is that EO material concepts that were developed for high poling effi

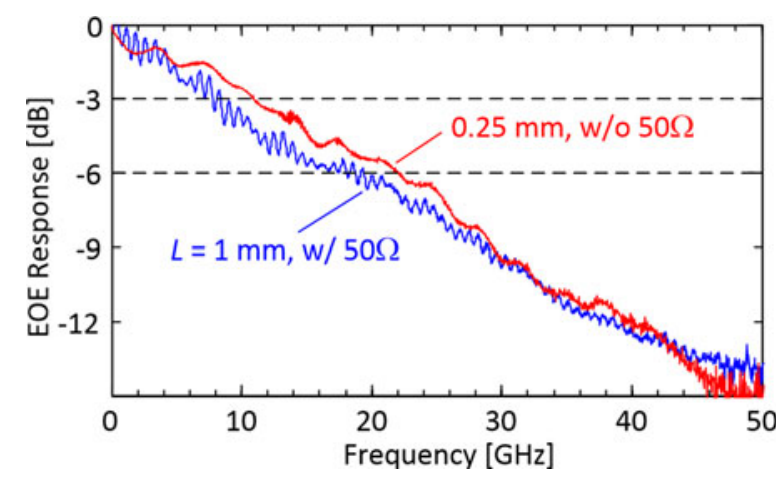

Fig. 5. Measured frequency response of a $1 \mathrm{~mm}$-long MZM with $50 \Omega$ termination (blue) and of a $250 \mu \mathrm{m}$-long non-terminated MZM. No gate voltage has been applied.

ciency in bulk materials also result in an increase of EO activity in SOH devices, as observed for the BCOG. Furthermore, the in-device $n^{3} r_{33}$ figur of merit $\left(U_{\pi} \propto\left(n^{3} r_{33}\right)^{-1}\right.$, see Eq. (1) of YLD124/PSLD41 is $1190 \mathrm{pm} / \mathrm{V}$ and is clearly superior to those of conventional EO materials such as GaAs $\left(\mathrm{n}^{3} r_{33} \approx 60\right.$ $\mathrm{pm} / \mathrm{V}[35])$ and $\mathrm{LiNbO}_{3}\left(<\mathrm{n}^{3} r_{33} \approx 400 \mathrm{pm} / \mathrm{V}[36]\right)$.

\section{DYNAMIC CHARACTERIZATION}

The measurements of the last section were performed at dc modulation voltages. To demonstrate that the increased EO coefficient can also be exploited at higher frequencies, we measure the frequency response of devices coated with the binary chromophore mixture YLD124/PSLD41 and perform data transmission experiments using on-off-keying (OOK) at data rates of up to $40 \mathrm{Gbit} / \mathrm{s}$.

\section{A. Frequency Response}

The frequency response is measured using a $\mathrm{CW}$ laser, a vector network analyzer (VNA), and a high-speed photodiode. The VNA was calibrated prior to the measurement in order to compensate for the frequency-dependent transfer function of the setup and the response of the photodiode. Fig. 5 depicts the measured electrical-optical-electrical (EOE) response of a $1 \mathrm{~mm}$-long modulator that is terminated by a $50 \Omega$ load (blue), and of a $250 \mu \mathrm{m}$-long non-terminated modulator (red). The $6 \mathrm{~dB}-E O E-b a n d w i d t h s$ are $18 \mathrm{GHz}$ and $22 \mathrm{GHz}$, respectively, and are comparable to those of samples that are coated with guest-host materials. We can therefore conclude that the bandwidth of the device is not detrimentally influence by the application of the BCOG. The bandwidth of the modulator could 

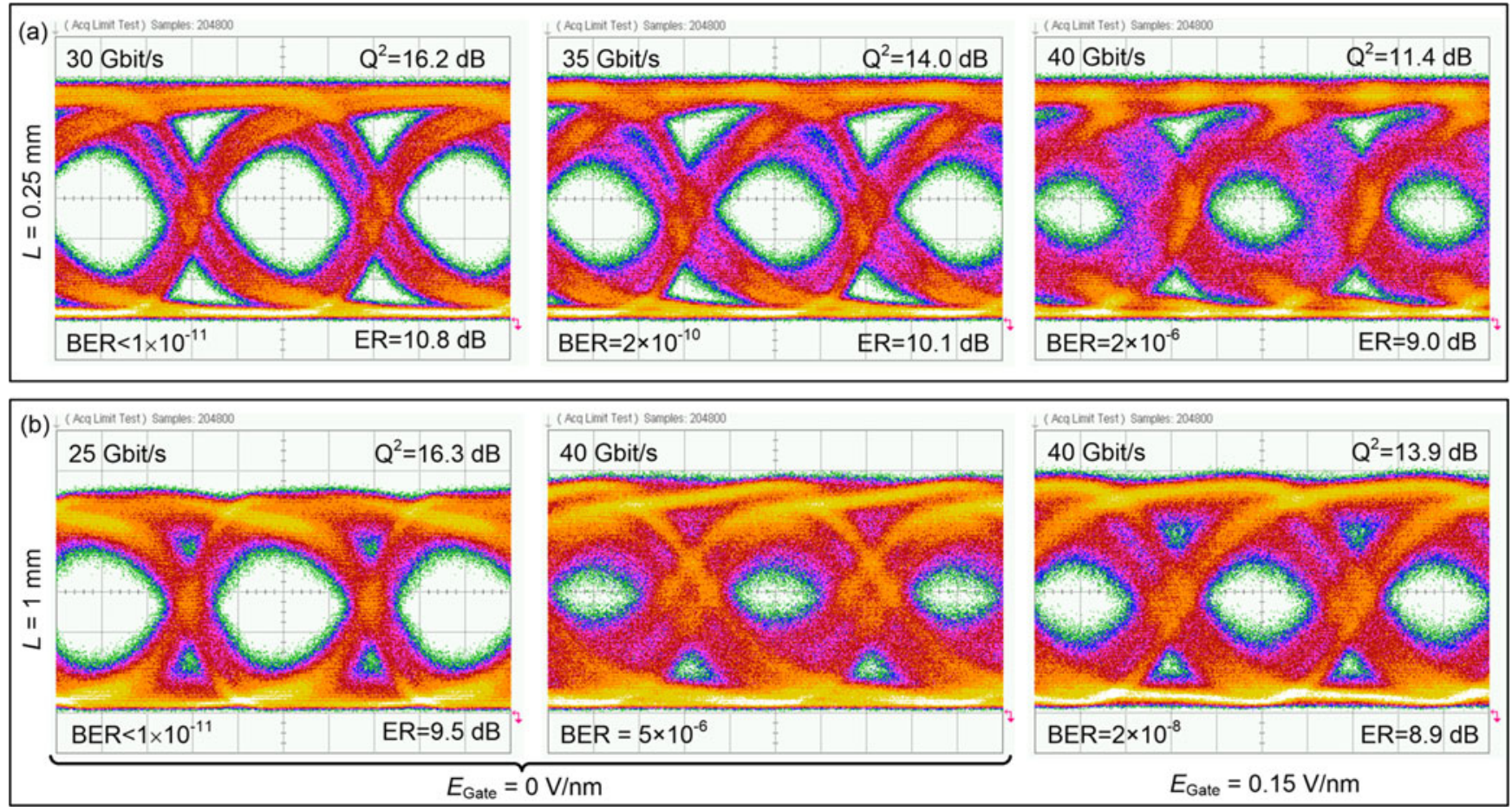

Fig. 6. NRZ OOK eye diagrams. Measured $\mathrm{Q}^{2}$-factors, extinction ratios (ER) and bit error ratios (BER) are denoted in the figures PRBS length $2^{31}-1$. (a) Operation at 30,35, and $40 \mathrm{Gbit} / \mathrm{s}$ obtained from a $250 \mu \mathrm{m}$-long MZM without termination. The drive voltage across the slot is $4.2 V_{\mathrm{pp}}$, the bias voltage amounts to $2.8 \mathrm{~V}$. The ER exceeds $10 \mathrm{~dB}$ up to $35 \mathrm{Gbit} / \mathrm{s}$. At $40 \mathrm{Gbit} / \mathrm{s}$ we measure a BER of $2 \times 10^{-6}$. (b) Operation at 25 and $40 \mathrm{Gbit} / \mathrm{s}$ obtained from a $1 \mathrm{~mm}$-long terminated MZM using a drive voltage of $1.5 V_{\mathrm{pp}}$. A low signal quality is achieved at $40 \mathrm{Gbit} / \mathrm{s}$ due to the small EO bandwidth of $18 \mathrm{GHz}$. Applying a gate fiel [11] of $0.15 \mathrm{~V} / \mathrm{nm}$ between the substrate and the transmission increases the bandwidth of the device from 18 to $25 \mathrm{GHz}$. This way the signal quality improves, and a BER of $2 \times 10^{-8}$ is measured at a data rate of $40 \mathrm{Gbit} / \mathrm{s}$.

be further improved by either increasing the doping concentration in the silicon slabs, by adding a second doping section of higher doping concentration close to the slot waveguide, or by applying a gate fiel that generates a highly conductive electron accumulation layer in the silicon slabs, see [11] for a more detailed discussion. A direct increase of the doping concentration to a value of $1 \times 10^{18} \mathrm{~cm}^{-3}$ in the slot waveguide section should boost the bandwidth to $27 \mathrm{GHz}$. At the same time the free-carrier absorption losses would increase from 1.3 to $1.9 \mathrm{~dB} / \mathrm{mm}$ [25], which corresponds to only a small increase of insertion loss by $0.6 \mathrm{~dB}$ for a $1 \mathrm{~mm}$-long MZM. For a $250 \mu \mathrm{m}$-long MZM, the loss penalty is less than $0.2 \mathrm{~dB}$.

\section{B. OOK Modulation}

Finally, we perform high-speed transmission experiments using a $250 \mu \mathrm{m}$-long non-terminated ("lumped") MZM and a $1 \mathrm{~mm}$-long terminated MZM, both coated with the mixture YLD124/PSLD41. First, the lumped MZM is investigated. Light from a tunable laser source at $1540 \mathrm{~nm}$ is coupled to the modulator. The device is connected to a pattern generator adjusted for a peak-to-peak voltage of $2.1 \mathrm{~V}_{\mathrm{pp}}$ if terminated with $50 \Omega$. Reflection of the RF wave at the end of the non-terminated device result in nearly a doubling of the in-device drive voltage to roughly $4.2 \mathrm{~V}_{\mathrm{pp}}$. A dc bias voltage of $2.8 \mathrm{~V}$ is combined with the RF signal by using a bias-T and the operation point of the MZM is set to the quadrature point. The modulated light is amplifie using an erbium doped fibe amplifie and received using a digital communications analyzer and a bit error ratio (BER) tester. Fig. 6(a) shows recorded eye diagrams of the $250 \mu \mathrm{m}$-long MZM for data rates ranging from 30 to $40 \mathrm{Gbit} / \mathrm{s}$. We observe excellent signal quality and extinction ratios (ER) exceeding $10 \mathrm{~dB}$ up to a data rate of $35 \mathrm{Gbit} / \mathrm{s}$. At $40 \mathrm{Gbit} / \mathrm{s}$ we measure a low BER of $2 \times 10^{-6}$ and an ER of $9 \mathrm{~dB}$.

Referring to [37] the energy consumption per bit $W_{\text {bit }}$ of the non-terminated MZM is given by

$$
W_{\mathrm{bit}}=\frac{1}{4} C_{\mathrm{d}} U_{\text {drive }}^{2}
$$

where $U_{\text {drive }}$ is the peak-to-peak drive voltage and $C_{d}$ is the device capacitance. The modulator has a measured capacitance of $95 \mathrm{fF}$ resulting in an energy consumption of $420 \mathrm{fJ} / \mathrm{bit}$. It is possible to reduce the energy consumption to a few fJ/bit by increasing the modulator length to $1 \mathrm{~mm}$ and simultaneously reducing the drive voltage below $400 \mathrm{~m} V_{\mathrm{pp}}$ as reported in [4]. Note that, in contrast to earlier demonstrations of $40 \mathrm{Gbit} / \mathrm{s}$ modulation in $\mathrm{SOH}$ devices [11], [14], we did not use a gate voltage to improve the silicon conductivity. Still, a small voltagelength product of $1 \mathrm{Vmm}$ is found for operation at $40 \mathrm{Gbit} / \mathrm{s}$, one order of magnitude below typical values reported for reversebiased pn-modulators [1], [5].

It should be noted that a slightly better $40 \mathrm{Gbit} / \mathrm{s}$ performance was reported in our previous work [38], where the initially small EO bandwidth of the device was increased from 10 to $25 \mathrm{GHz}$ by applying a gate voltage between transmission line and silicon substrate. In this paper, $40 \mathrm{Gbit} / \mathrm{s}$ operation was achieved 
without the use of a gate voltage. However, the comparison to [38] indicates that the doping concentration and the doping profil need to be further optimized for high-speed operation.

For the $1 \mathrm{~mm}$-long MZM the drive voltage is set to $1.5 \mathrm{~V}_{\mathrm{pp}}$. Recorded eye diagrams are depicted in Fig. 6(b). Without the application of a gate voltage [11], the measured $40 \mathrm{Gbit} / \mathrm{s}$ eye diagram is hardly open, since the EO bandwidth of this device is only $18 \mathrm{GHz}$. In analogy to our previous work in [38] we can increase this bandwidth to $25 \mathrm{GHz}$ by applying a gate fiel of $0.15 \mathrm{~V} / \mathrm{nm}$. This clearly improves the quality of the $40 \mathrm{Gbit} / \mathrm{s}$ eye diagram and leads to an ER of $9 \mathrm{~dB}$ and a BER of $2 \times 10^{-8}$.

\section{CONCLUSION AND OUTLOOK}

We demonstrate that EO coefficient as high as $230 \mathrm{pm} / \mathrm{V}$ can be achieved in $\mathrm{SOH}$ devices that use structurally engineered electro-optic organic materials. Using a binary-chromophore organic glass composed of the chromophores YLD124 and PSLD41, we demonstrate an SOH MZM with small voltagelength product of $0.52 \mathrm{Vmm}$ measured at dc. This concept enables $250 \mu \mathrm{m}$ long non-terminated modulators suitable for operation at $40 \mathrm{Gbit} / \mathrm{s}$.

For a practical application of $\mathrm{SOH}$ devices, the long-term stability of the organic cladding is of high importance. The glass transition temperature of the investigated BCOG is relatively low $\left(T_{g}=97^{\circ} \mathrm{C}\right)$, resulting in thermally activated re-orientation of the chromophores. One month after poling we measure a degradation of the EO coefficien by $20 \%$. The investigation of aging and temperature stability of various organic materials is subject to ongoing research. We expect that material stability can be significantl improved by synthetically modifie chromophores that bear specifi crosslinking agents for postpoling lattice hardening or by increasing the molar mass of the chromophores. The viability of the firs approach has already been demonstrated for similar EO compounds [39], [40], where material stability of up to $250{ }^{\circ} \mathrm{C}$ has been achieved.

\section{ACKNOWLEDGMENT}

Peter Johnston (University of Washington) is acknowledged for the synthesis of YLD124.

\section{REFERENCES}

[1] D. J. Thomson, F. Y. Gardes, Y. Hu, G. Mashanovich, M. Fournier, P. Grosse, M. Fedeli, and G. T. Reed, "High contrast 40Gbit/s optical modulation in silicon," vol. 19, no. 12, pp. 11507-11516, 2011.

[2] W. M. Green, M. J. Rooks, L. Sekaric, and Y. A. Vlasov, "Ultra-compact, low RF power, $10 \mathrm{~Gb} / \mathrm{s}$ silicon Mach-Zehnder modulator," Opt. Exp., vol. 15, no. 25, pp. 17106-17113, 2007.

[3] J. Fujikata, J. Ushida, T. Nakamura, Y. Ming-Bin, Z. ShiYang, D. Liang, P. L. Guo-Qiang, and D.-L. Kwong, " $25 \mathrm{GHz}$ operation of silicon optical modulator with projection MOS structure," presented at the Opt. Fiber Commun. Conf., San Diego, CA, 2010, Paper OMI3.

[4] D. J. Thomson, F. Y. Gardes, J.-M. Fedeli, S. Zlatanovic, Y. Hu, B. P. P. Kuo, E. Myslivets, N. Alic, S. Radic, G. Z. Mashanovich, and G. T. Reed, "50-Gb/s silicon optical modulator," IEEE Photon. Technol. Lett., vol. 24, no. 4, pp. 234-236, Feb. 2012.

[5] G. T. Reed, G. Mashanovich, F. Y. Garde, and D. J. Thomson, "Silicon optical modulators," Nature Photon., vol. 4, pp. 518-526, Aug. 2010.

[6] T. Baehr-Jones, R. Ding, Y. Liu, A. Ayazi, T. Pinguet, N. C. Harris, M. Streshinsky, P. Lee, Y. Zhang, A. E.-J. Lim, T.-Y. Liow, S. H.-G. Teo, G.-Q. Lo, and M. Hochberg, "Ultralow drive voltage silicon travelingwave modulator," Opt. Exp., vol. 20, no. 11, pp. 12014-12020, 2012.
[7] M. R. Watts, W. A. Zortman, D. C. Trotter, R. W. Young, and A. L. Lentine, "Vertical junction silicon microdisk modulators and switches," Opt. Exp., vol. 19, no. 22, pp. 21989-22003, 2011.

[8] J. Leuthold, W. Freude, J.-M. Brosi, R. Baets, P. Dumon, I. Biaggio, M. L. Scimeca, F. Diederich, B. Frank, and C. Koos, "Silicon organic hybrid technology - A platform for practical nonlinear optics," Proc. IEEE, vol. 97, no. 7, pp. 1304-1316, Jul. 2009.

[9] T. W. Baehr-Jones and M. J. Hochberg, "Polymer silicon hybrid systems: A platform for practical nonlinear optics," J. Phys. Chem. C, vol. 112, no. 21, pp. 8085-8090, May 2008 .

[10] J.-M. Brosi, Slow-Light Photonic Crystal Devices for High-Speed Optical Signal Processing. Karlsruhe, Germany: University Univ. of Karlsruhe, 2008.

[11] L. Alloatti, D. Korn, R. Palmer, D. Hillerkuss, J. Li, A. Barklund, R. Dinu, J. Wieland, M. Fournier, J. Fedeli, H. Yu, W. Bogaerts, P. Dumon, R. Baets, C. Koos, W. Freude, and J. Leuthold, "42.7 Gbit/s electro-optic modulator in silicon technology," Opt. Exp., vol. 19, no. 12, pp. 11841-11851, Jun. 2011.

[12] T. Baehr-Jones, B. Penkov, J. Huang, P. Sullivan, J. Davies, J. Takayesu, J. Luo, T.-D. Kim, L. Dalton, A. Jen, M. Hochberg, and A. Scherer, "Nonlinear polymer-clad silicon slot waveguide modulator with a half wave voltage of 0.25 V," Appl. Phys. Lett., vol. 92, no. 16, p. 163303, 2008. Doi: 10.1063/1.2909656..

[13] R. Palmer, L. Alloatti, D. Korn, P. C. Schindler, M. Baier, J. Bolten, T. Wahlbrink, M. Waldow, R. Dinu, W. Freude, C. Koos, and J. Leuthold, "Low power Mach-Zehnder modulator in silicon-organic hybrid technology," IEEE Photon. Technol. Lett., vol. 25, no. 13, pp. 1226-1229, Jul. 2013.

[14] R. Palmer, L. Alloatti, D. Korn, P. C. Schindler, R. M. Schmogrow, M. Baier, S. Koenig, D. Hillerkuss, J. Bolten, T. Wahlbrink, M. Waldow, R. Dinu, W. Freude, C. Koos, and J. Leuthold, "Silicon-organic hybrid (SOH) modulator generating up to 84 gbit/s BPSK and M-ASK signals," presented at the Opt. Fiber Commun. Conf./Nat. Fiber Opt. Eng. Conf., Anaheim, CA, 2013, Paper OW4J.6.

[15] R. Palmer, S. Koeber, W. Heni, D. L. Elder, D. Korn, H. Yu, L. Alloatti, S. Koenig, P. C. Schindler, W. Bogaerts, M. Pantouvaki, G. Lepage, P. Verheyen, J. Van Campenhout, P. Absil, R. Baets, L. R. Dalton, W. Freude, J. Leuthold, and C. Koos, "High-speed silicon-organic hybrid (SOH) modulator with $1.6 \mathrm{fJ} / \mathrm{bit}$ and $180 \mathrm{pm} / \mathrm{V}$ in-device nonlinearity," in Proc. 39th Eur. Conf. Exhib. Opt. Commun., 2013, pp. 510-512.

[16] R. Ding, T. Baehr-Jones, Y. Liu, R. Bojko, J. Witzens, S. Huang, J. Luo, S. Benight, P. Sullivan, J.-M. Fedeli, M. Fournier, L. Dalton, A. Jen, and M. Hochberg, "Demonstration of a low V pi L modulator with $\mathrm{GHz}$ bandwidth based on electro-optic polymer-clad silicon slot waveguides," Opt. Exp., vol. 18, no. 15, pp. 15618-15623, 2010.

[17] D. L. Elder, S. J. Benight, J. Song, B. H. Robinson, and L. R. Dalton, "Matrix-assisted poling of monolithic bridge-disubstituted organic NLO chromophores," Chem. Mater., vol. 26, pp. 872-874, Jan. 2014.

[18] J. Takayesu, M. Hochberg, T. Baehr-Jones, E. Chan, P. Sullivan, J. Davies, L. Dalton, A. Scherer, and W. Krug, "A hybrid electrooptic microring resonator-based $1 \times 4 \times 1$ ROADM for wafer scale optical interconnects," J. Light. Technol., vol. 27, no. 4, pp. 440-448, Feb. 2009.

[19] X. Wang, C.-Y. Lin, S. Chakravarty, J. Luo, A. K.-Y. Jen, and R. T. Chen, "Effective in-device r33 of $735 \mathrm{pm} / \mathrm{V}$ on electro-optic polymer infil trated silicon photonic crystal slot waveguides," Opt. Lett., vol. 36, no. 6, pp. 882-884, 2011.

[20] M. Gould, T. Baehr-Jones, R. Ding, S. Huang, J. Luo, A. K.-Y. Jen, J.-M. Fedeli, M. Fournier, and M. Hochberg, "Silicon-polymer hybrid slot waveguide ring-resonator modulator.," Opt. Exp., vol. 19, no. 5, pp. 39523961, 2011.

[21] V. R. Almeida, Q. F. Xu, C. A. Barrios, and M. Lipson, "Guiding and confinin light in void nanostructure," Opt. Lett., vol. 29, no. 11, pp. 12091211, Jun. 2004.

[22] D. Korn, R. Palmer, H. Yu, P. C. Schindler, L. Alloatti, M. Baier, R. Schmogrow, W. Bogaerts, S. K. Selvaraja, G. Lepage, M. Pantouvaki, J. M. D. Wouters, P. Verheyen, J. Van Campenhout, B. Chen, R. Baets, P. Absil, R. Dinu, C. Koos, W. Freude, and J. Leuthold, "Silicon-organic hybrid (SOH) IQ modulator using the linear electro-optic effect for transmitting 16QAM at $112 \mathrm{Gbit} / \mathrm{s}, "$ Opt. Exp., vol. 21, no. 11, pp. 1321913227, Jun. 2013

[23] R. Palmer, L. Alloatti, D. Korn, W. Heni, P. C. Schindler, J. Bolten, M. Karl, M. Waldow, T. Wahlbrink, W. Freude, C. Koos, and J. Leuthold, "Low-loss silicon strip-to-slot mode converters," Photon. J., vol. 5, no. 1, 2013. Doi: 10.1109/JPHOT.2013.2239283. 
[24] D. Taillaert, F. Van Laere, M. Ayre, W. Bogaerts, D. Van Thourhout, P. Bienstman, and R. Baets, "Grating couplers for coupling between optical fiber and nanophotonic waveguides," Jpn. J. Appl. Phys., vol. 45, no. 8A, pp. 6071-6077, 2006.

[25] R. A. Soref and B. R. Bennett, "Electrooptical effects in silicon," IEEE J. Quantum Electron., vol. 23, no. 1, pp. 123-129, Jan. 1987.

[26] S. Selvaraja, M. Gayle, M. Alexey, D. Christie, O. Patrick, S. Pathak, D. Vermeulen, G. Sterckx, G. Winroth, P. Verheyen, G. Lepage, R. Baets, W. Bogaerts, J. Van Campenhout, and P. Absil, "Advanced 300-mm waferscale patterning for silicon photonics devices with record low loss and phase errors," presented at the17th OptoElectron. Commun. Conf., Busan, Korea, 2012, Paper PDP2-2.

[27] R. Ding, T. Baehr-Jones, W.-J. Kim, X. Xiong, R. Bojko, J.-M. Fedeli, M. Fournier, and M. Hochberg, "Low-loss strip-loaded slot waveguides in silicon-on-insulator," Opt. Exp., vol. 18, no. 24, pp. 25061-25067, Nov. 2010.

[28] R. Ding, T. Baehr-Jones, W.-J. Kim, B. Boyko, R. Bojko, A. Spott, A. Pomerene, C. Hill, W. Reinhardt, and M. Hochberg, "Low-loss asymmetric strip-loaded slot waveguides in silicon-on-insulator," Appl. Phys. Lett., vol. 98, p. 233303, 2011. Doi: 10.1063/1.3597798.

[29] L. R. Dalton, D. Lao, B. C. Olbricht, S. Benight, D. H. Bale, J. A. Davies, T. Ewy, S. R. Hammond, and P. A. Sullivan, "Theory-inspired development of new nonlinear optical materials and their integration into silicon photonic circuits and devices," Opt. Mater., vol. 32, no. 6, pp. 658-668, Apr. 2010.

[30] Y. V. Pereverzev, K. N. Gunnerson, O. V. Prezhdo, P. A. Sullivan, Y. Liao, B. C. Olbricht, A. J. P. Akelaitis, A. K.-Y. Jen, and L. R. Dalton, "Guesthost cooperativity in organic materials greatly enhances the nonlinear optical response," J. Phys. Chem. C, vol. 112, no. 11, pp. 4355-4363, Mar. 2008.

[31] P. A. Sullivan, H. Rommel, Y. Liao, B. C. Olbricht, A. J. P. Akelaitis, K. A. Firestone, J.-W. Kang, J. Luo, J. A. Davies, D. H. Choi, B. E. Eichinger, P. J. Reid, A. Chen, A. K.-Y. Jen, B. H. Robinson, and L. R. Dalton, "Theory-guided design and synthesis of multichromophore dendrimers: An analysis of the electro-optic effect," J. Amer. Chem. Soc., vol. 129 , no. 24, pp. 7523-7530, Jun. 2007.

[32] P. A. Sullivan and L. R. Dalton, "Theory-inspired development of organic electro-optic materials," Acc. Chem. Res., vol. 43, no. 1, pp. 10-8, Jan. 2010.

[33] J. Witzens, T. Baehr-Jones, and M. Hochberg, "Design of transmission line driven slot waveguide Mach-Zehnder interferometers and application to analog optical links," Opt. Exp., vol. 18, no. 16, pp. 16902-16928, 2010.

[34] Y. Enami, C. T. Derose, D. Mathine, C. Loychik, C. Greenlee, R. A. Norwood, T. D. Kim, J. Luo, Y. Tian, A. K.-Y. Jen, and N. Peyghambarian, "Hybrid polymer/sol-gel waveguide modulators with exceptionally large electro-optic coefficients, Nature Photon., vol. 1, no. 3, pp. 180-185, Mar. 2007.

[35] N. Dagli, "Ultralow drive voltage substrate removed GAAS/ALGAAS electro-optic modulators at $1550 \mathrm{~nm}$," IEEE J. Sel. Toics Quantum Electron., vol. 19, no. 6, pp. 150-157, Nov. 2013.

[36] R. W. Boyd, Nonlinear Optics, 3rd ed. New York, NY, USA: Academic, 2008.

[37] D. A. B. Miller, "Energy consumption in optical modulators for interconnects," Opt. Exp., vol. 20, no. S2, pp. A293-A308, 2012.

[38] R. Palmer, L. Alloatti, D. Korn, P. C. Schindler, R. Schmogrow, W. Heni, S. Koenig, J. Bolten, T. Wahlbrink, M. Waldow, H. Yu, W. Bogaerts, P. Verheyen, G. Lepage, M. Pantouvaki, J. Van Campenhout, P. Absil, R. Dinu, W. Freude, C. Koos, and J. Leuthold, "Silicon-organic hybrid MZI modulator generating OOK, BPSK and 8-ASK signals for up to 84 Gbit/s," IEEE Photon. J., vol. 5, no. 2, pp. 6600907-6600907, Apr. 2013.

[39] J. Luo, S. Huang, Z. Shi, B. M. Polishak, X.-H. Zhou, and A. K. Jen, "Tailored organic electro-optic materials and their hybrid systems for device applications," Chem. Mater, vol. 23, no. 3, pp. 544-553, Feb. 2011.

[40] Z. Shi, W. Liang, J. Luo, S. Huang, B. M. Polishak, X. Li, T. R. Younkin, B. a. Block, and A. K.-Y. Jen, "Tuning the kinetics and energetics of dielsalder cycloaddition reactions to improve poling efficien y and thermal stability of high-temperature cross-linked electro-optic polymers," Chem. Mater., vol. 22, no. 19, pp. 5601-5608, Oct. 2010.
Robert Palmer received the Diploma degree in physics ("with distinction") from the Karlsruhe Institute of Technology (KIT), Karlsruhe, Germany. Since 2010, he has been a Research Associate at the Institute of Photonics and Quantum Electronics, KIT, Karlsruhe, Germany, where he is currently working toward the Ph.D. degree in electrical engineering. His research interests include integrated optics and silicon-organic hybrid integration. In 2013, he received the Corning Outstanding Student Paper Award (firs prize) at the Optical Fiber Communication Conference and the ADVA Best Student Paper Award (firs prize) at the European Conference on Optical Communication.

Sebastian Koeber received the Ph.D. degree in physical chemistry from Cologne University, Germany, in 2011. Since 2012, he has been a Postdoctoral Researcher and Research Group Leader at the Institute of Photonics and Quantum Electronics and Institute of Microstructure Technology, both at the Karlsruhe Institute of Technology. His research interests include silicon-organic hybrid integration, organic nonlinear optical materials, and optical biosensors based on polymers.

Delwin L. Elder received the B.S. degree in chemistry from the University of North Carolina, Chapel Hill, NC, USA, in 1994 and the Ph.D. degree from the California Institute of Technology, in 1999. From 1999 to 2009, he was a Senior Research Chemist at Air Products and Chemicals, Inc., where his research and development projects included polymer electrolytes for lithium batteries and charge transport conducting polymers, among other things. Since 2010, he has been a Research Scientist with the Chemistry Department, University of Washington, Seattle, WA, USA. His research interests include synthesis of organic nonlinear optical chromophores and characterization of electro-optic coefficient physical properties, and bulk molecular order.

Markus Woessner received the B.Sc. degree in electrical engineering from the Karlsruhe Institute of Technology, Karlsruhe, Germany, in 2013. His thesis focused on "Novel Organic Materials for Electro-Optic Devices in Silicon." $\mathrm{He}$ is currently working toward the M.Sc. degree in Optics and Photonics, Karlsruhe Institute of Technology.

Wolfgang Heni received the M.Sc. degree in electrical engineering ("with distinction") from the Karlsruhe Institute of Technology in 2013. In his thesis he investigated organic electrooptic switches and modulators in silicon. $\mathrm{He}$ is currently working toward the Ph.D. degree in electrical engineering at ETH Zurich, Zurich, Switzerland. He is a Research Associate at the Electromagnetic Fields and Microwave Electronics Laboratory (IFH), ETH Zurich. His research interests include integrated photonic and plasmonic devices.

Dietmar Korn received the Diploma degree in physics from the University of Potsdam, Potsdam, Germany. From 2009 to 2013, he was a Research Associate at the Institute of Photonics and Quantum Electronics, Karlsruhe Institute of Technology, Karlsruhe, Germany, from where he received the Ph.D. degree in electrical engineering in 2013. He is currently working at Imagine Optic SA, France. 
Matthias Lauermann received the Diploma degree from the Department of Electrical Engineering and Information Technology, Karlsruhe Institute of Technology, Germany, in 2011, where he is currently working toward the Ph.D. degree. He is a Research Associate at the Institute of Photonics and Quantum Electronics and affiliate with the Helmholtz International Research School for Teratronics. His research interests include hybrid silicon photonic systems for optical communication and Terabit/s signals within the scope of the EU-FP7 project PhoxTroT.

Wim Bogaerts (S'98-M'05) received the M.Sc. degree in engineering (applied physics) from Ghent University, Gent, Belgium, in 1998, and the Ph.D. degree in electrical engineering (photonics) in 2004. He is currently a Professor of silicon photonics at the Photonics Research Group of Ghent University - IMEC. He specializes in the design challenges for large-scale photonic integration. During the Ph.D. research and subsequent postdoctoral work he realized the firs demonstrations of silicon photonics at IMEC. This work also led to the multiproject wafer service ePIXfab. Since 2010, he has been a Professor at Ghent University. He has been, or is, actively involved in the European projects PICCO, PICMOS, ePIXnet, WaDiMOS, HELIOS, SOFI, and Plat4M. In 2014, he cofounded the start-up Luceda with a focus on photonic-electronic design automation, in which he is involved on a part-time basis. He is a Member of IEEE Photonics Society, the Optical Society of America, and SPIE.

Larry Dalton (SM'06) received the B.S. degree from the Honors College of Michigan State University, East Lansing, MI, USA, in 1965 and the Ph.D. degree from Harvard University, Cambridge, MA, USA, in 1971. He is currently the B. Seymour Rabinovitch Chair Professor (Emeritus) and the George B. Kauffman University Professor (Emeritus) of Chemistry and Electrical Engineering, University of Washington, Seattle, WA, USA. He is the Founding Director of the National Science Foundation Science and Technology Center on Materials and Devices for Information Technology Research. He is a Fellow of the American Chemical Society, the Materials Research Society, the Optical Society of America, the SPIE International Society of Optics and Photonics, and the American Association for the Advancement of Science. He received the 2007 IEEE/LEOS William Streifer Scientifi Achievement Award, the 2003 ACS Chemistry of Materials National Award, the 2011 ACS Linus Pauling Award and Medal, the 1996 ACS Richard C. Tolman Award and Medal, and the SPIE Lifetime Achievement Award (2008).
Wolfgang Freude received the Dipl.-Ing. (M.S.E.E.) and the Dr.-Ing. (Ph.D.E.E.) degrees in electrical engineering in 1969 and 1975 from the University of Karlsruhe, Karlsruhe, Germany, respectively.

$\mathrm{He}$ is currently a Professor at the Institute of Photonics and Quantum Electronics and a Distinguished Senior Fellow at Karlsruhe Institute of Technology. His research activities include the area of optical high-data rate transmission, high-density integrated-optics with a focus on silicon photonics, photonic crystals and semi-conductor optical amplifiers and in the fiel of low-energy opto-electronic devices and protocols for optical access networks. He has published more than 240 papers, coauthored a book entitled "Optical Communications" (Berlin, Germany, Springer-Verlag, 1991, in German), and authored and co-authored four book chapters on "Multimode Fibres" in Handbook of Optical Communications (Berlin, Germany: Springer-Verlag, 2002, in German), "Microwave Modelling of Photonic Crystals" in Photonic CrystalsAdvances in Design, Fabrication, and Characterization (Berlin, Germany: Wiley-VCH, 2004), "Linear Semiconductor Optical Amplifiers in Fibre Optic Communication - Key Devices, Berlin, Germany: Springer-Verlag, 2012, and "Optical OFDM and Nyquist Multiplexing" in Optical Fiber Telecommunications VI B. Systems and Networks (Amsterdam, Elsevier, 2013).

Prof. Freude is an Honorary Doctor of the Kharkov National University of Radioelectronics, Kharkov, Ukraine, and a Member of VDE/ITG, and OSA. He serves in the Technical Program Committee "Photonic Networks and Devices" (OSA Advanced Photonics Congress 2013 and 2014), and was the Chair of the Subcommittee "Micro- and Nano-Photonic Devices" for CLEO 2012 and 2013. Until 2010, he was the Vice Chair of the IEEE Germany Photonics Society Chapter. From 2002 to 2006, he served as the Spokesman of the Research Training Group "Mixed Fields and Nonlinear Interactions," which was funded by the Deutsche Forschungsgemeinschaft (DFG) to support young researchers in their pursuit of a doctorate.

Juerg Leuthold received the Ph.D. degree in physics from ETH Zurich, Zurich, Switzerland, in 1998. From 1999 to 2004, he was with Bell Labs, Lucent Technologies in Holmdel, USA, where he performed device and system research. From July 2004 to February 2013, he was a Full Professor at the Karlsruhe Institute of Technology, where he was the Head of the Institute of Photonics and Quantum Electronics and the Head of the Helmholtz Research Institute of Micro Structure Technology. Since March 2013, he has been a Full Professor at ETH Zurich. He is a Fellow of the Optical Society of America.

Christian Koos received the Dipl.-Ing. (M.Sc.) and Dr.-Ing. (Ph.D.) degrees in electrical engineering from the University of Karlsruhe, Karlsruhe, Germany, in 2002 and 2007, respectively. From 2007 to 2008, he carried out postdoctoral research at the Institute of Photonics and Quantum Electronics (IPQ), University of Karlsruhe. From 2008 to 2010, he was with the Corporate Research and Technology Department, Carl Zeiss AG, Germany, where he led the technology forecast in area of nanotechnology. Since 2010, he has been a Full Professor at Karlsruhe Institute of Technology, where he is currently heading the IPQ and the Institute of Microstructure Technology. 